Research Paper

\title{
Comparing Survival between Peritoneal Dialysis and Hemodialysis Patients with Subclinical Peripheral Artery Disease: a 6-Year Follow-Up
}

\author{
Jiung-Hsiun Liu',2,3, Jan-Yow Chen 2,4, Shih-Yi Lin ${ }^{1}$, Hsin-Hung Linn',2, I-Wen Ting1, Chih-Chia Liang1, \\ I-Kuan Wang1,2, Huey-Liang Kuo1,2, Chiz-Tzung Chang1,2, Chiu-Ching Huang1,2,*凶, Fung-Chang Sung3,* \\ 1. Division of Nephrology and Kidney Institute, Department of Internal Medicine, China Medical University Hospital, Taichung, Taiwan \\ 2. School of Medicine, College of Medicine, China Medical University, Taichung, Taiwan. \\ 3. Department of Public Health, College of Public Health, China Medical University, Taichung, Taiwan. \\ 4. Division of Cardiology, Department of Internal Medicine, China Medical University Hospital, Taichung, Taiwan. \\ ${ }^{*}$ Equal contributors.
}

$\square$ Corresponding author: Chiu-Ching Huang, MD. Division of Nephrology and Kidney Institute, China Medical University Hospital, 2 Yu-Der Road, Taichung 404, Taiwan. E-mail: cch@mail.cmuh.org.tw.

(0) Ivyspring International Publisher. This is an open-access article distributed under the terms of the Creative Commons License (http://creativecommons.org/ licenses/by-nc-nd/3.0/). Reproduction is permitted for personal, noncommercial use, provided that the article is in whole, unmodified, and properly cited.

Received: 2012.08.23; Accepted: 2013.02.25; Published: 2013.03.0I

\begin{abstract}
Peripheral artery disease (PAD) is known to be an increased mortality risk in patients with end-stage renal disease (ESRD). The aim of this study was to compare patient survival between patients with subclinical PAD undergoing peritoneal dialysis (PD) and hemodialysis (HD). Subclinical peripheral artery was defined as an ankle-brachial index of less than 0.9. This study was conducted from April 2005, and the observation period ended on 30 June 20II. At the end of the follow-up, the status of all patients was assessed and data on mortality were obtained for the entire cohort. A total of 91 patients (6I HD and $30 \mathrm{PD}$ ) were included for analyses in this study. Mortality rate was $60.0 \%$ (I8/30) for PD and 52.5\% (32/6I) for HD. Kaplan-Meier estimate demonstrate that PD patients had a higher mortality rate than those underwent HD (log-rank $p=0.0039)$. Cox regression model demonstrated that $\mathrm{PD}$ was an independent predictor for further mortality in ESRD patients with subclinical peripheral artery disease. $(\mathrm{p}=0.012, \mathrm{HR}$ : $1.776,95 \% \mathrm{Cl}$ : I.136-2.775). In multivariate analysis, the HD group still had a greater survival than PD group ( $\mathrm{P}=$ 0.005 , HR:I.916, $95 \% \mathrm{Cl}: 1.218-3.015)$. In patients with subclinical peripheral artery disease, the patient survival is better in HD patients as compared with PD patients.
\end{abstract}

Key words: Survival, hemodialysis, peritoneal dialysis, peripheral artery disease.

\section{Introduction}

End-stage renal disease (ESRD) is a powerful determinant of atherosclerotic vascular disease and is associated with a remarkably high incidence of cardiovascular morbidity. The presence of cardiovascular disease is an important predictor of mortality in patients with ESRD, as it accounts for almost 40 percent of deaths [1]. Peripheral arterial disease (PAD) is an important manifestation of systemic atherosclero- sis and is common among ESRD patients. It is growing numbers of patients are now with PAD as a result of the trend of increased prevalence of ESRD in the past decade [2]. Epidemiological and clinical studies of the ESRD patients have clearly shown that PAD is a strong predictor for subsequent mortality $[3,4]$. Notably, the relative risk of PAD for overall mortality is similar to that of preexisting coronary artery disease, 
congestive heart failure and celebrovascular disease in ESRD patients [5]. Thus, it has recently attracted much attention of PAD as a risk factor for adverse outcomes in ESRD patients.

It is undoubtedly the hemodialysis (HD) and peritoneal dialysis (PD) to serve the patients with ESRD as the most common methods for renal replacement therapy worldwide. The general consensus is that HD and PD can be viewed as equivalent therapies and either modality may be used as primary therapy for patients with new ESRD [6]. The merits of mortality differences between PD and HD have been widely debated over the past decade. Several studies comparing mortality between PD and HD among ESRD patients show conflicting and inconsistent results [5, 7-12]. The discrepancy of these results may result from methodological difference, period of follow-up and stratified subgroup [13]. Therefore, if we want to know whether there are survival benefits of one modality compared with the other; we may not to answer the question in specific high-risk subgroups with ESRD.

It is not a rare situation to encounter chronic kidney disease or dialysis patients with PAD. The prevalence of overt or subclinical PAD was counted approximately $20-30 \%$ in patients receiving dialysis treatment $[3,14,15]$. Previous comparisons between PD and HD patients have not fully compared survival outcomes in high-risk subgroup, especially in those with preexisting cardiovascular disease. PD patients are exposed to greater amounts of daily glucose loading, leading to a much higher prevalence of insulin resistance, dyslipidemia and metabolic syndrome [16]. There is also evidence that PD patients exhibit greater coagulability status [17]. PD may also accelerate development of atherosclerosis lesions via increased glycosylation and lipid oxidation $[18,19]$. In contrast, HD patients are exposed to a greater risk of cardiovascular disease due to more rapid decline of residual renal function [20] and more hyperdynamic status conferred on the presence of the arteriovenous fistula and extracorporeal circulation [21]. Since the PAD is a marker of systemic atherosclerosis [22], the impact of the dialysis modality on the mortality in patients with PAD remains unclear. The aim of the study presented here was to investigate the effects of dialysis modality on overall mortality in an ESRD population with subclinical PAD.

\section{Methods}

\section{Study design and patients}

During the period from April 2005 to September 2005, a total of 421 patients (153 PD and 268 HD) un- derwent maintenance renal replacement therapy was included for screen of subclinical PAD at China Medical University Hospital in Taiwan. All patients who fulfilled the following inclusion criteria were considered for enrollment into this study: (i) patients who had received regular dialysis treatment at least for six months; (ii) patients' modality of renal replacement therapy was the initial type without using another types before; (iii) patients had to be clinically stable for three months before entry, without infectious or other active diseases. (iv) patients met the criteria for diagnosis of subclinical PAD according to the reports of ankle-brachial blood pressure index (ABI). The enrolled ESRD patients for screen of subclinical PAD included 179 males and 242 females. The dialysis regimen and frequency were prescribed by each patient's attending nephrologist. Clinical indications were the main reasons for change in dialysis regimen and frequency. The study was approved by institutional review board of the hospital and all enrolled patients gave written, informed consent.

\section{Data collection}

Clinical information was obtained by hospital records and dialysis logs. Blood sample was drawn in the morning after overnight fast and demographic information were included as confounders at study baseline. Blood pressure was measured in the arm after $20 \mathrm{~min}$ of rest in the supine position with a standard mercury sphygmomanometer, and the auscultatory method was used with cuff-size adjustment based on arm circumference at study baseline. Pulse pressure was calculated for each individual as the difference between systolic blood pressure and diastolic blood pressure. The preexisting cardiovascular disease (CVD) was defined as patients who had history of angina, coronary disease, myocardial infarction, abnormal angiographic results, transient ischemic attack, and/or cerebrovascular accident. Diabetes was defined as a previous physician diagnosis, or fasting glucose of more than $126 \mathrm{mg} / \mathrm{dL}(7.0$ $\mathrm{mmol} / \mathrm{L})$, or nonfasting glucose of more than 200 $\mathrm{mg} / \mathrm{dL}(11.1 \mathrm{mmol} / \mathrm{L})$ at baseline. Anuria was defined as a status of completely anuric without passage of urine amount in study subjects.

\section{$A B I$ measurement and definition of subclinical PAD}

The value of $\mathrm{ABI}$ was measured using an ABI-form device (VP1000; Coli, Komaki, Japan), which automatically and simultaneously measures blood pressures in both arms (brachial arteries) and ankles (posterior tibial arteries) by using an oscillometric method. The ABI was calculated by dividing 
systolic blood pressure, measured at the ankle, by systolic blood pressure, measured at the arm. Subclinical PAD was defined as an ABI value less than 0.90 in either extremity.

\section{Endpoint and outcome analyses}

Clinical outcomes in this study included actual patient survival. Patients transferred to alternative renal replacement therapies were censored at the time of transfer. Also, data for patients who were lost to follow-up were censored for the survival analysis. The observation period ended on 30 June 2011. At the end of the follow-up, the status of all patients assessed and data on mortality were obtained for the entire cohort.

\section{Statistical analysis}

Categorical data were expressed as absolute numbers or percentages and the $\chi^{2}$-test was applied for comparison. Continuous data were expressed as mean \pm standard deviation for normally distributed data, and as median and interquartile range for nonparametric data. The Student's t-test was used when data were normally distributed; otherwise, the non-parametric Mann-Whitney U-test was used. Survival curves were generated using the Kaplan-Meier technique and tested using the log-rank test. Variables predictive of all-cause mortality were determined using Cox regression models. Any selected variable for which there was a significant difference for prediction of mortality in univariate Cox models was considered as a risk factor and entering into the multivariate Cox model. Hazard ratios (HRs) and 95\% confidence intervals (CIs) were measured for variable included. A p-value less than 0.05 was considered as statistically significant. All calculations were performed with the SPSS software version 12.0 for Windows (SPSS Institute, Chicago, IL).

\section{Results}

\section{Patient characteristics}

From April 2005 to September 2005, we identified 91 patients ( 29 males and 62 females; 61 on HD and 30 on PD) with ESRD and subclinical PAD. The prevalence of subclinical PAD was $21.6 \%(91 / 421)$ at the study baseline in our patients. Table 1 shows the characteristics of the patients' cohort at baseline by dialysis modality. The HD group had a longer mean duration of dialysis $(\mathrm{p}=0.04)$, a higher mean pre-dialysis blood urea nitrogen $(p=0.001)$, and a lower mean serum triglyceride level $(p=0.006)$. The HD group was also more prevalent with diabetes $(\mathrm{p}=$ $0.004)$.

Table I. Clinical characteristics of the patients with end-stage renal disease with subclinical artery disease who were underwent hemodialysis or peritoneal dialysis.

\begin{tabular}{llll}
\hline & Hemodialysis $(\mathrm{n}=61)$ & Peritoneal dialysis $(\mathrm{n}=30)$ & P value \\
\hline Age (yrs) & $63.8 \pm 11.4$ & $64.3 \pm 11.2$ & 0.82 \\
Gender (male/female) & $20 / 41$ & $9 / 21$ & 0.789 \\
Duration of dialysis (months) & $54(19-83)$ & $28.5(17.8-58.3)$ & 0.04 \\
Diabetes (yes/no) & $40 / 21$ & $10 / 20$ & 0.004 \\
Ankle-brachial index & $0.76 \pm 0.20$ & $0.76 \pm 0,17$ & 0.786 \\
Systolic blood pressure (mmHg) & $124.7 \pm 29.3$ & $129.5 \pm 30.2$ & 0.084 \\
Diastolic blood pressure (mmHg) & $76.7 \pm 13.0$ & $80.3 \pm 18.2$ & 0.102 \\
Pulse pressure (mmHg) & $46.9 \pm 19.7$ & $47.3 \pm 16.5$ & 0.325 \\
BUN (mg/dL) & $74.7 \pm 23.2$ & $58.8 \pm 17.9$ & 0.001 \\
Creatinine (mg/dL) & $10.6 \pm 2.1$ & $11.2 \pm 2.6$ & 0.262 \\
Intact parathyroid hormone (pg/mL) & $156(62.4-360)$ & $84.5(27.6-433)$ & 0.498 \\
Preexisting CVD (\%) & $26(42.6)$ & $12(40.0)$ & 0.811 \\
Cholesterol (mg/dL) & $181.7 \pm 50.4$ & $197.4 \pm 56.3$ & 0.186 \\
Triglyceride (mg/dL) & $145(79-250.5)$ & $244(139.5-410.5)$ & 0.006 \\
Hematocrit (\%) & $29.6 \pm 3.8$ & $28.6 \pm 4.0$ & 0.236 \\
Calcium (mg/dL) & $9.0 \pm 0.8$ & $9.3 \pm 1.1$ & 0.151 \\
Phosphate (mg/dL) & $5.7 \pm 1.3$ & $5.3 \pm 1.3$ & 0.303 \\
Albumin (g/dL) & $3.5 \pm 0.4$ & $3.7 \pm 0.4$ & 0.084 \\
\hline
\end{tabular}




\section{Causes of death in relation to dialysis modality}

During the study period, 50 patients (32 HD and 18 PD) died. The causes of death (Figure 1) in the HD group were sudden death in 9 patients $(28.1 \%)$, cardiovascular disease in 7 patients $(21.9 \%)$, celebrovascular disease in 3 patients $(9.4 \%)$, infection with sepsis in 9 patients $(28.1 \%)$, malignancies in 3 patients $(9.4 \%)$, and other miscellaneous cause in 1 patient $(3.1 \%)$. The causes of death (Figure 1) in the PD group were sudden death in 1 patient $(5.6 \%)$, cardiovascular disease in 1 patient $(5.6 \%)$, celebrovascular disease in 3 patients (16.7\%), unresolved PD related peritonitis in 6 patients $(33.3 \%)$, infection with sepsis in 2 patients $(11.1 \%)$, and other miscellaneous causes in 5 patients $(27.8 \%)$. The overall cardiovascular cause (sudden death, cardiovascular disease and celebrovascular disease) of death was $48.0 \%$. HD patients had a higher mortality from cardiovascular causes than PD group (59.4\% vs $27.8 \%, \mathrm{p}<0.05)$.

\section{Patient survival in relation to dialysis modality}

During the study period, if patients were censored from the survival analysis, his or her health status was considered as the health status at the time of censor. After counting the events of death in other patients, the remaining patients were administratively censored on 30 June, 2011. Figure 2 shows the comparison of survival curves for ESRD patients with subclinical PAD between those on HD and PD. The curves show a statistically significant difference between the two group $(p=0.0039)$. Patient survival in the group on PD was lower compared with those on
HD. The increased mortality was particularly evident after the first 12 months of follow-up.

To further examine the relationship between dialysis modality and patient's mortality, each potential risk factor was entered into the adjusted-mortality model. To further examine the relationship between dialysis modality and patient mortality, each potential risk factor was entered into the adjusted-mortality model. The analysis indicated that older age was an independent factor positively associated mortality (HR, 1.033 [95\% CI, 1.013-1.053]). Compared with the HD group, the PD group was at higher risk of mortality (HR, 1.916 [95\% CI, 1.218-3.015]).

\section{Patient survival in PD patients with and with- out residual renal function}

In the PD group, 19 patients were noted to be completely anuric and 11 patients were not at study baseline. As comparison of survival between patients on HD and PD without anuric, the mortality difference gap was decreased $(p=0.0533)$ (Figure was not shown).

There was interesting trend regarding the impact of residual renal function on survival. Patients with anuric had an increased risk of all-cause mortality compared to non-anuric patients. The increased mortality was particularly evident in the first 40-45 months, leveling off thereafter despite of no statistical significance was identified (Figure 3). The result provide a trend that the mortality difference is more likely related to residual renal function of PD patients at the study entry.

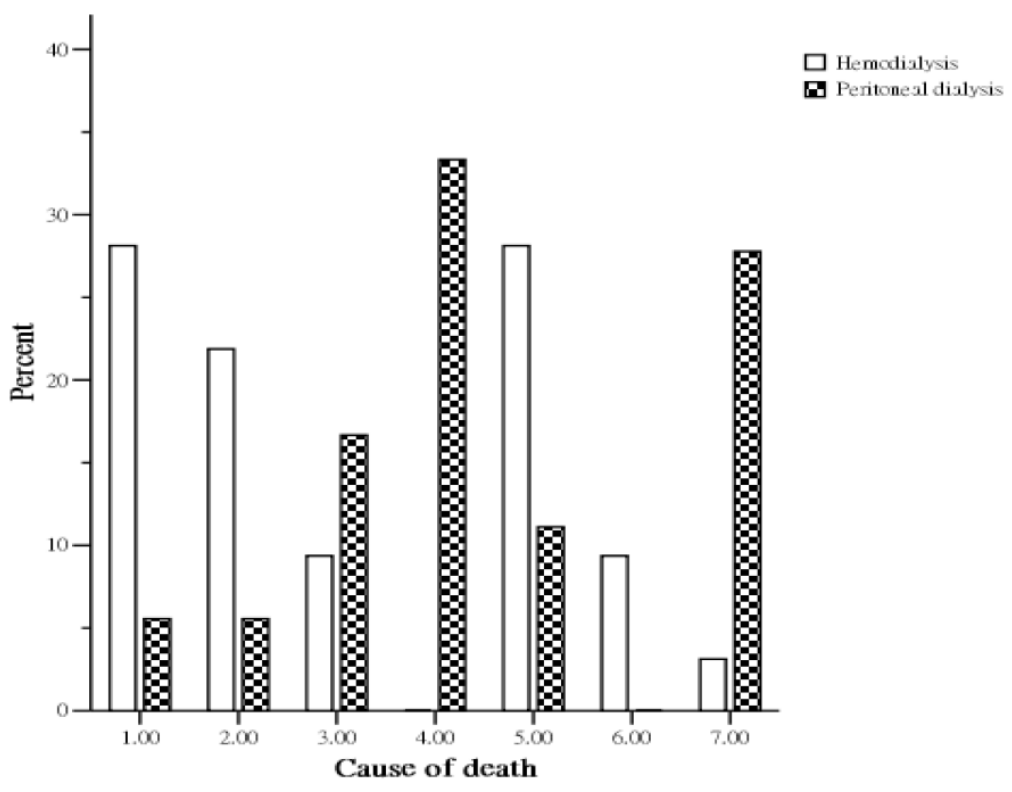

Fig I. Percent distribution of mortality by cause between hemodialysis and peritoneal dialysis patients. $(I=$ sudden death; $2=$ cardiovascular disease; $3=$ celebrovasuclar disease; $4=$ peritoneal dialysis associated peritonitis; $5=$ other infections; $6=$ malignancy; $7=$ miscellaneous causes). 


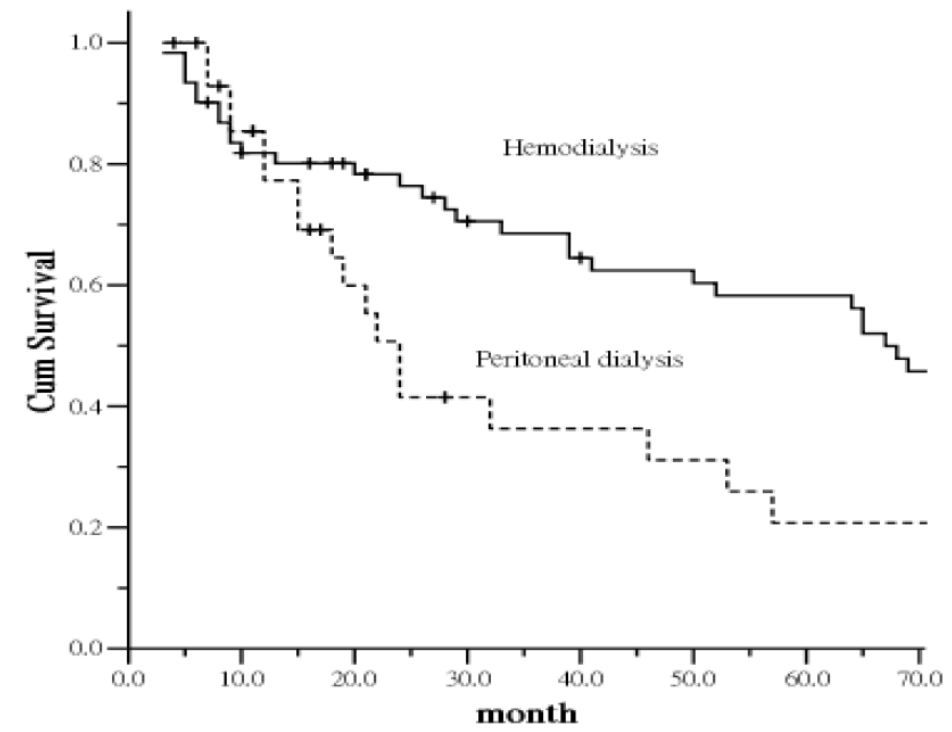

Fig 2. Kaplan-Meier plot of survival curve in relation to dialysis modality.

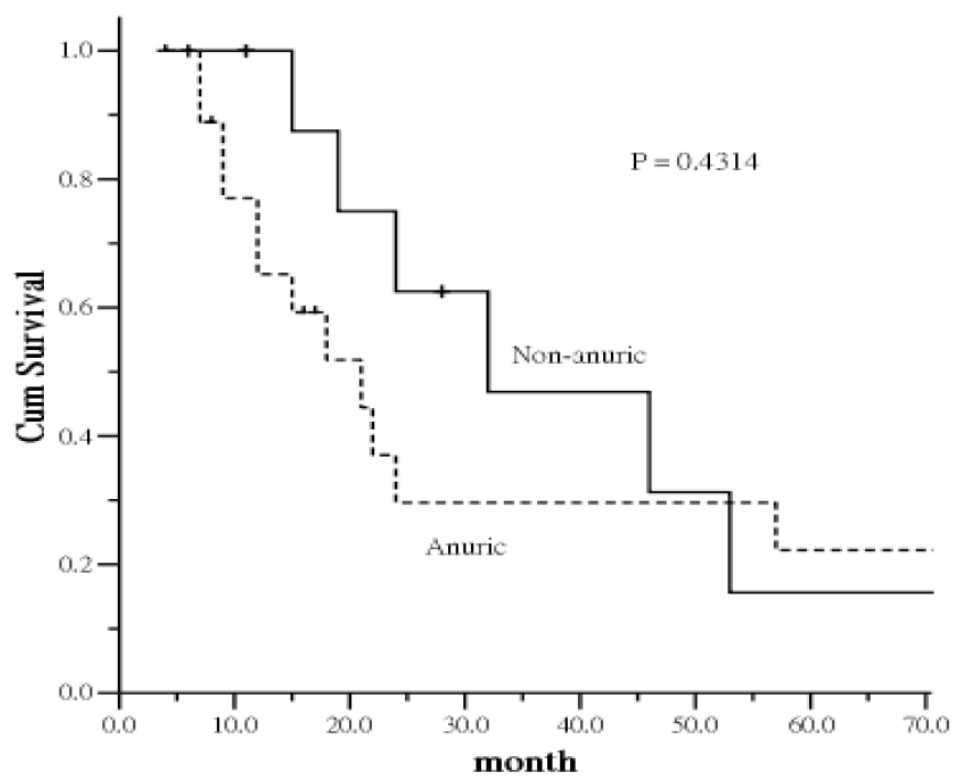

Fig 3. Kaplan-Meier plot of survival curve in relation to anuric status in peritoneal dialysis patients.

Table 2. Mortality risk associated with risk factors in univariate and multivariate Cox proportional hazards models.

\begin{tabular}{|c|c|c|c|c|}
\hline Variable & $\begin{array}{l}\text { Hazard ratio } \\
\text { (95\% confidence interval) }\end{array}$ & $\mathrm{P}$ & $\begin{array}{l}\text { Adjusted hazard ratio (95\% } \\
\text { confidence interval) }\end{array}$ & $\mathrm{P}$ \\
\hline Age (per 1 yr increase) & $1.031(1.011-1.051)$ & 0.002 & $1.033(1.013-1.053)$ & 0.001 \\
\hline Gender (male vs female) & $1.413(0.906-2.205)$ & 0.127 & - & \\
\hline Dialysis modality (PD vs HD) & $1.776(1.136-2.775)$ & 0.012 & $1.916(1.218-3.015)$ & 0.005 \\
\hline Preexisting CVD & $1.241(0.814-1.241)$ & 0.315 & - & \\
\hline Duration of dialysis (per 1 month increase) & $1.003(0.998-1.007)$ & 0.264 & - & \\
\hline Diabetes & $0.902(0.595-1.367)$ & 0.626 & - & \\
\hline BUN (per 1 mg/dL increase) & $0.997(0.989-1.006)$ & 0.577 & - & \\
\hline Triglyceride (per 1 mg/dL increase) & $1.000(0.999-1.001)$ & 0.492 & - & \\
\hline Albumin (per $1 \mathrm{~g} / \mathrm{dL}$ increase) & $0.702(0.371-1.328)$ & 0.277 & - & \\
\hline
\end{tabular}




\section{Discussion}

Choosing the right patient for the right modality has been shown to have a great impact not only on health-related outcomes but on quality of life at the time of initial dialysis. The question of which dialysis modality would cause lesser further mortality to ESRD patients with a history of PAD is encountered frequently preceded and underwent dialysis in clinical practice. Give that cardiac mortality is the greatest cause to all-cause mortality in the ESRD and PAD populations [23]; vigorous efforts are required to identify factors that may exacerbate this problem. This study presented here demonstrated that the risk of death was significantly increased in PD patients compared with HD patients with subclinical PAD. Our findings are consistent with finding in previous studies $[5,8,12]$, which has suggested poorer outcomes in PD patients with underlying cardiovascular diseases.

The increased mortality risk among subclinical PAD patients treated with PD was not constant over time but increased with follow-up. The increased mortality in PD was particularly evident after the first 12 months of follow-up. This finding suggests that the deleterious effect of PD on the survival of ESRD patients with subclinical PAD is a time-dependent phenomenon. The development of associated mechanism leading to increased mortality may be a function of time as well. Previous studies comparing PD- and HD-treated patients rarely compared survival outcomes in high-risk subgroups, especially in those with preexisting cardiovascular disease. Given that cardiovascular disease is prevalent in diagnosed ESRD patients, defining the optimal dialysis modality for patients would reduce further morbidity and mortality.

Our nonrandomized observational study demonstrates a significant difference in the risk of mortality between patients on PD and HD. The dialysis modality may interact with subclinical PAD associated with mortality. This study does not, however, provide evidence for the mechanism of increased mortality among PD-treated patients. There are, however, several possibilities. First, the importance of residual renal function has become evidence in PD patients in the past decade. Decline of residual renal function is associated with fluid overload [24], anemia [25], inflammation [26], malnutrition [27], PD-related peritonitis [28], and mortality [29,30] among PD patients. In contrast, the importance of reservation of residual renal function is playing lesser crucial role in influencing further survival among HD patients. Second, PAD patients have a high prevalence of con- comitant left ventricular hypertrophy in patients without ESRD at first diagnosis. Otherwise, a cross-sectional survey demonstrates the greater preservation of residual renal function, the lesser prevalence of left ventricular hypertrophy in PD patients [31]. Therefore, left ventricular hypertrophy is to potentially make a major contribution to cardiac death in PAD patients [32]. Prospective comparisons of PD and HD patients have shown significantly more left ventricular hypertrophy and poorer left ventricular function among PD-treated patients. These studies implied the possibility of mechanism of impact of residual renal function on mortality risk in PD patients with PAD [33,34]. Finally, patients treated with PD have a higher percentage of death due to infection than those with $\mathrm{HD}$ in present study $(44.4 \%$ vs. $28.1 \%)$. It has been well documented that innate immunity is not only a key element in host to defense against bacterial infections but also associated with atherosclerosis and cardiovascular diseases [35-39]. Macrophage scavenger receptor has a crucial role in innate immunity system, and it has the integral membrane protein containing a collagenous domain associated with pathological deposition of cholesterol during atherogenesis as a result of uptake of modified low-density lipoproteins. Macrophage knockout mice exhibit a marked decrease in modified low-density lipoproteins uptake in vitro and are more susceptible to infection. The effect of protection from infection by innate immunity system is probable impairment in patients on PD as compared with HD [40].

There are several limitations in this study. First, the number of study subjects is modest, and could not set complex models with confounding factors in multivariate analyses. A larger cohort of patients is needed to confirm our findings. Second, our included subjects were not newly diagnosed dialysis patients, and we can therefore not study the precise effect of modality from incident time. Third, our study was based on ABI value to define PAD, which was subclinical rather than overt PAD. The definition of overt PAD probably needs an angiography. However, the calculation of ABI values is a non-invasive and convenient measure currently available surrogate marker as it presented good validation for detecting PAD. Finally, the degree of residual renal function was not quantified in our PD patients at study baseline. Thus, we were unable to evaluate whether there is dose-response relationship between residual renal function and PAD. Further examination for this relationship is necessary.

\section{Conclusion}

In summary, our findings suggest that $\mathrm{PD}$, as is 
currently practiced, may not be a suitable choice for ESRD patients with PAD and that that HD is a preferred therapy. Given the epidemic of atherosclerosis and alarmingly high prevalence of PAD in ESRD patients, there is an urgent need for a large size prospective study in this population. Further study is also needed to explore the accurate mechanism on the mortality discrepancy between PD and HD in this population.

\section{Author Contribution}

Chiu-Ching Huang and Fung-Chang Sung designed the research; Jiung-Hsiun Liu, Shih-Yi Lin, Hsin-Hung Lin, I-Wen Ting, Chih-Chia Liang, Huey-Liang Kuo, Chiz-Tzung Chang and I-Kuan Wang performed the research; Jiung-Hsiun Liu and Shih-Yi Lin analyzed the data; Jiung-Hsiun Liu and Jan-Yow Chen developed the manuscript.

\section{Conflict of Interest}

The authors report no conflicts of interest.

\section{References}

1. United States Renal Data System: Annual Data Report. Am J Kidney Dis 2012;59(Suppl 1):S225-32.

2. Rajagopalan S, Dellegrottaglie S, Furniss AL, et al. Peripheral arterial disease in patients with end-stage renal disease: observations from the Dialysis Outcomes and Practice Patterns Study (DOPPS). Circulation 2006;114:1914-22.

3. Liu JH, Lin HH, Yang YF, et al. Subclinical peripheral artery disease in patients undergoing peritoneal dialysis: risk factors and outcome. Perit Dial Int 2009;29:64-71.

4. Ono K, Tsuchida A, Kawai H, Matsuo H, et al. Ankle-brachial blood pressure index predicts all-cause and cardiovascular mortality in hemodialysis patients. J Am Soc Nephrol 2003;14:1591-8.

5. Stack AG, Molony DA, Rahman NS, Dosekun A, Murthy B. Impact of dialysis modality on survival of new ESRD patients with congestive heart failure in the United States. Kidney Int 2003;64:1071-9.

6. Gokal R. Peritoneal dialysis in the $21^{\text {st }}$ century: An analysis of current problems and further developments. J Am Soc Nephrol 2002;13(Suppl 1):S104-16.

7. Collins AJ, Hao W, Xia H, et al. Mortality risks of peritoneal dialysis and hemodialysis. Am J Kidney Dis 1999;34:1065-74

8. Ganesh SK, Hulbert-Shearon T, Port FK, Eagle K, Stack AG. Mortality differences by dialysis modality among incident ESRD patients with and without coronary artery disease. J Am Soc Nephrol 2003;14:415-24.

9. Weinhandl ED, Foley RN, Gilbertson DT, Arneson TJ, Snyder JJ, Collins AJ. Propensity-matched Mortality comparison of incident hemodialysis and peritoneal dialysis patients. J Am Soc Nephrol 2010;21:499-506.

10. Vonesh EF, Snyder JJ, Foley RN, Collins AJ. The differential impact of risk factors on mortality in hemodialysis and peritoneal dialyisis. Kidney Int 2004;66:2389-401.

11. McDonald SP, Marshall MR, Johnson DW, Polkinghorne KR: Relationship between dialysis modality and mortality. J Am Soc Nephrol 2009;20:155-63.

12. Johnson DW, Dent H, Hawley CM, et al. Association of dialysis modality and cardiovascular mortality in incident dialysis patients. Clin J Am Soc Nephrol 2009;4:1620-8

13. Vonesh EF, Snyder JJ, Foley RN, Collins AJ. Mortality studies comparing peritoneal dialysis and hemodialysis, what do they tell us? Kidney Int 2006;70 (Suppl 103 ): S3-11.

14. Liu JH, Chang CC, Wang SM, et al. Peripheral arterial disease and clinical risks in Taiwanese hemodialysis patients. Angiology 2010;61:66-73.

15. O'Hare AM, Hsu CY, Bacchetti P, Fohansen KL. Peripheral vascular disease risk factors among patients undergoing hemodialysis. J Am Soc Nephrol 2002;13:497-503.
16. Johnson DW, Armstrong K, Campbell B, et al. Metabolic syndrome in severe chronic kidney disease: prevalence, predictors, prognostic significance and effects of risk factor modification. Nephrology 2007;12:391-8.

17. Kobayashi M, Yorioka N, Yamakido M. Hypercoagulability and secondary hyperfibrinolysis may be related to abnormal lipid metabolism in patients treated with continuous ambulatory peritoneal dialysis. Nephron 1997;76:56-61.

18. Kimak E, Solski J, Janicka L, Ksaziek A, Janicki K. Concentration of Lp(a) and other apolipoproteins in predialysis, hemodialysis, chronic ambulatory peritoneal dialysis and post-transplant patients. Clin Chem Lab Med 2000;38:421-5.

19. Miyata T, Sugiyama S, Saito A, Kurokawa K. Reactive carbonyl compounds related uremic toxicity ("carbonyl stress"). Kidney Int 2001;78: S25-31.

20. Moist LM, Port FK, Orzol SM, et al. Predictors of loss of residual renal function among new dialysis patients. J Am Soc Nephrol 2000;11:556-64.

21. Dikow R, Schwenger V, Zeier M, Ritz E. Do AV fistulas contribute to cardiac mortality in hemodialysis patients? Semin Dial 2002;15:14-7.

22. Hirsch AT, Criqui MH, Treat-Jacobson D, et al. Peripheral arterial disease detection, awareness, and treatment in primary care. JAMA 2001;286:1317-24.

23. Criqui MH, Langer RD, Fronek A, et al. Mortality over a period of 10 years in patients with peripheral arterial disease. $\mathrm{N}$ Engl J Med 1992;326:381-6.

24. Ateş K, Nergizoğlu G, Keven K, et al. Effect of fluid and sodium removal on mortality in peritoneal dialysis patients. Kidney Int 2001;60:767-76.

25. Wang AY, Wang M, Woo J, et al. A novel association between residual renal function and left ventricular hypertrophy in peritoneal dialysis patients. Kidney Int 2002;62:639-47.

26. Pecoits-Filho R, Heimbürger O, Bárány $\mathrm{P}$, et al. Associations between circulating inflammatory markers and residual renal function in CRF patients. Am J Kidney Dis 2003;41:1212-8.

27. Wang AY, Woo J, Wang M, et al. Important differentiation of factors that predict outcome in peritoneal dialysis patients with different degrees of residual renal function. Nephrol Dial Transplant 2005;40:396-403.

28. Han SH, Lee SC, Ahn SV, et al. Reduced residual renal function is a risk of peritonitis in continuous ambulatory peritoneal dialysis patients. Nephrol Dial Transplant 2007;22:2653-8.

29. Bargman JM, Thorpe KE, Churchill DN. Relative contribution of residual renal function and peritoneal clearance to adequacy of dialysis: a reanalysis of the CANUSA study. J Am Soc Nephrol 2001;12:2158-62.

30. Maiorca R, Brunori G, Zubani R, et al. Predictive value of dialysis adequacy and nutritional indices for mortality and morbidity in CAPD and HD patients. A longitudinal study. Nephrol Dial Transplant 1995;10:2295-305.

31. Wang AY, Wang M, Woo J, et al. A novel association between residual renal function and left ventricular hypertrophy in peritoneal dialysis patients. Kidney Int 2002;62:639-47.

32. Wright GA, Ang DS, Stonebridge PA, Belch JJ, Struthers AD. Left ventricular hypertrophy is present in one-half of newly-diagnosed peripheral arterial disease patients. J Hyperts 2007;25:463-9.

33. Enia G, Mallamaci F, Benedetto FA, et al. Long-term CAPD patients are volume expanded and display more severe left ventricular hypertrophy than haemodialysis patients. Nephrol Dial Transplant 2001;16:1459-64.

34. Takeda K, Nakamoto M, Hirakata H, Baba M, Kubo M, Fujishima M. Disadvantage of long-term CAPD for preserving cardiac performance: an echocardiographic study. Am J Kidney Dis 1998;32:482-7.

35. Suzuki H, Kurihara $Y$, Takeya $M$, et al. A role for macrophage scavenger receptors in atherosclerosis and susceptibility to infection. Nature 1997;386:292-6.

36. Suzuki H, Kurihara Y, Takeya M, et al. The multiple roles of macrophage scavenger receptors (MSR) in vivo: resistance to atherosclerosis and susceptibility to infection in MSR knockout mice. J Atheroscler Thromb 1997;4:1-11.

37. Kato S, Chmielewski M, Honda $\mathrm{H}$, et al. Aspects of immune dysfunction in end-stage renal disease. Clin J Am Soc Nephrol 2008;3:1526-33.

38. de Winther MP, van Dijk KW, Havekes LM, Hofker MH. Macrophage scavenger receptor class A: A multifunctional receptor in atherosclerosis. Arterioscler Thromb Vasc Biol 2000;20:290-7.

39. Thomas CA, Li Y, Kodama T, et al. Protection from lethal gram-positive infection by macrophage scavenger receptor-dependent phagocytosis. J Exp Med 2000;191:147-56.

40. Ando M, Shibuya A, Yasuda M, et al. Impairment of innate cellular response to in vitro stimuli in patients on continuous ambulatory peritoneal dialysis. Nephrol Dial Transplant 2005;20:2497-503. 